16 ADAPTABILITY OF 'MODEL FOR IMPROVEMENT' CONTRIBUTION TO MATERNAL AND NEWBORN CARE IN THE LEARNING NETWORK (PROTOTYPE DISTRICT, BANGLADESH)

${ }^{1}$ Minara Chowdhury, ${ }^{2}$ Muntasir Moin, ${ }^{3}$ Zubair Shams. 'Institute for Healthcare Improvement, UK; ${ }^{2}$ Institute for Healthcare Improvement, Bangladesh; ${ }^{3}$ Save the Children, Bangladesh

\subsection{6/bmjoq-2020-|HI.16}

Background Bangladesh has adopted the WHO framework on Maternal and Newborn care. USAID's MaMoni MNCSP works to improve maternal and newborn care across the public and private health systems. Project baseline study found about poor health facility readiness (3\%) for quality antenatal care, $9 \%$ of facilities had partograph available, and about $20 \%$ of providers were trained on ANC and delivery care. In 2019, Model for Improvement (MFI), a method for structuring an improvement project was introduced. Altogether, facilities implemented and tested Maternal Newborn Health (MNH) clinical bundles comprising 73 improvement projects on quality ANC, correct partograph use, (CPU) and Essential Newborn Care (ENC) using the PDCA approach. A visual display board reflect facility performance and periodic review.

Objectives To describe the effectiveness of 'Model for Improvement' in improving $\mathrm{MNH}$ clinical bundles under learning network model.

Methods Manikganj district was selected as project intervention district. A total of 31 diversified facilities has joined this network to learn about improvement methodologies and its implication. The 'MFI" framework were used for continuous improvement. This model helped defined their aim, ideas, and measurement process. Also the PDCA (Plan Do Check Act) cycle outlined the steps for the actual testing of the change ideas. A comparative analysis of selected $\mathrm{MNH}$ indicators was done between baseline survey (2019) and service statistics (2020).

Results About 200 health managers and service providers were trained on different clinical themes and leadership. Supportive supervision was deployed. Thus resulted improvement in quality ANC from $20 \%$ to $85 \%$, CPU from $26 \%$ to $95 \%$ and quality ENC from $9 \%$ to $86 \%$.

Conclusions Such initiative in Bangladesh has facilitated innovation both within and across facilities. This network of care can rapidly achieve significant improvements in quality health care. With results from Manikganj, project is scaling up $\mathrm{MNH}$ clinical bundles in other districts.

\section{Featured posters}

\section{STANDARDIZING STROKE CARE ACROSS THE CONTINUUM: LEARNINGS FROM A NATIONAL COLLABORATIVE}

Heather Forst. Vizient, USA

\subsection{6/bmjoq-2020-IHI.17}

Background One of the biggest challenges we face in health care is minimizing variations in care across the continuum. Vizient created a national collaborative focused on stroke which identified leading strategies in reducing variations in stroke care system-wide. According to the Centers for Disease Control and Prevention, each year more than 795,000 people in the United States have a stroke; and health system's direct medical costs are projected to reach $\$ 94.3$ billion by 2035. In terms of market impact, stroke and neurovascular disease represent a significant portion of inpatient volumes and source of growth- representing 34\% of US market discharges in 2018.

Objectives In 2019, fifty organizations comprised of large academic medical centers, community health and critical access hospitals participated in Vizient's Stroke Collaborative to network with peers across the nation to learn leading strategies and share improvement information. Leading subject matter experts shared knowledge and discussed current standards of stroke care, identified innovative ways to improve care transitions and assessed care settings to optimize delivery of patient care across the continuum.

Methods May - October 2019. Two distinct cohorts were developed for primary stroke and comprehensive stroke centers who met bi-monthly for education, coaching and networking opportunities. Collaborative Requirements: Complete project charter, monthly progress updates, baseline and remeasure data submissions.

Results Over a 6 month time period, collaborative participants measured 30-day readmissions to measure improvement across

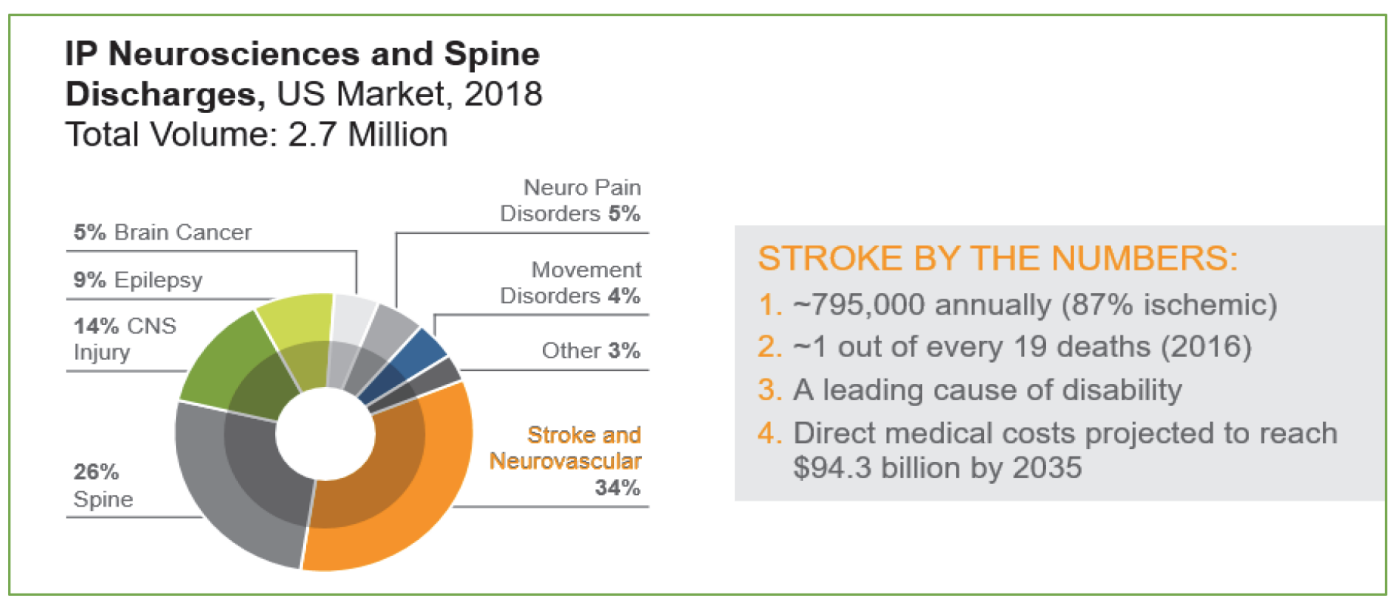

Abstract 17 Figure 1 Stroke volumes and disease burden in the US from Sg2 analytics 
\% Point Difference 30-day Readmissions Rate

Baseline (April 1 - Sept. 30, '18) to Remeasure (April 1 - Sept. 30, '19)

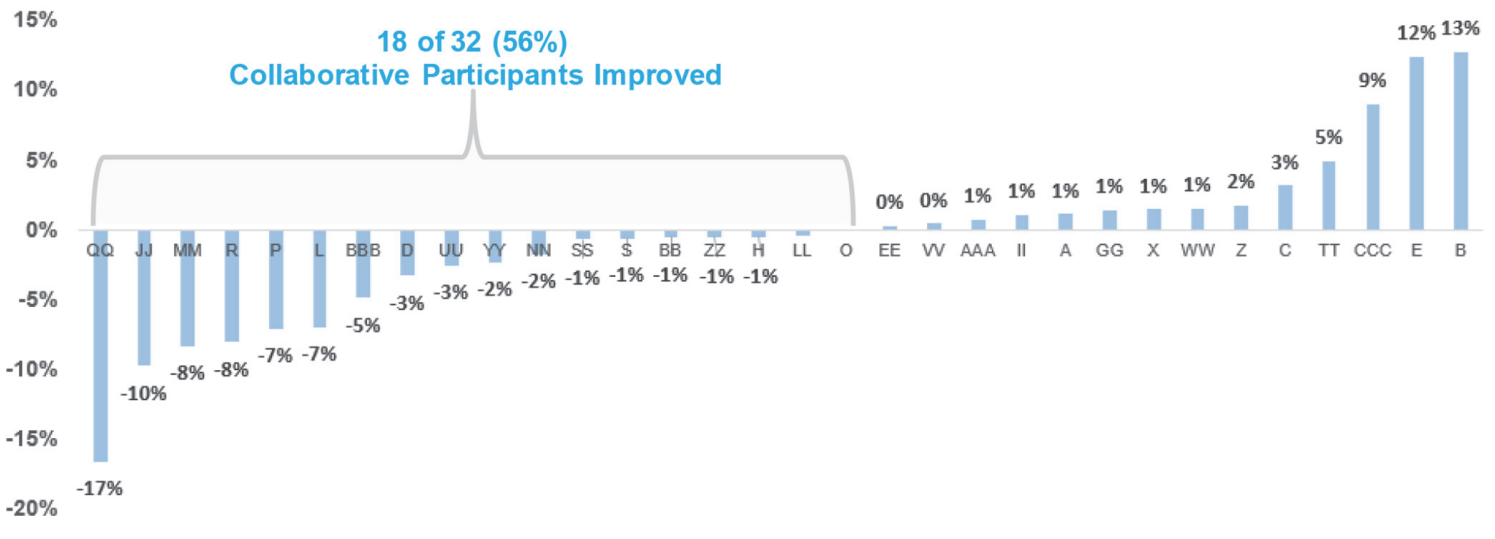

Abstract 17 Figure 2 Ischemic stroke performance

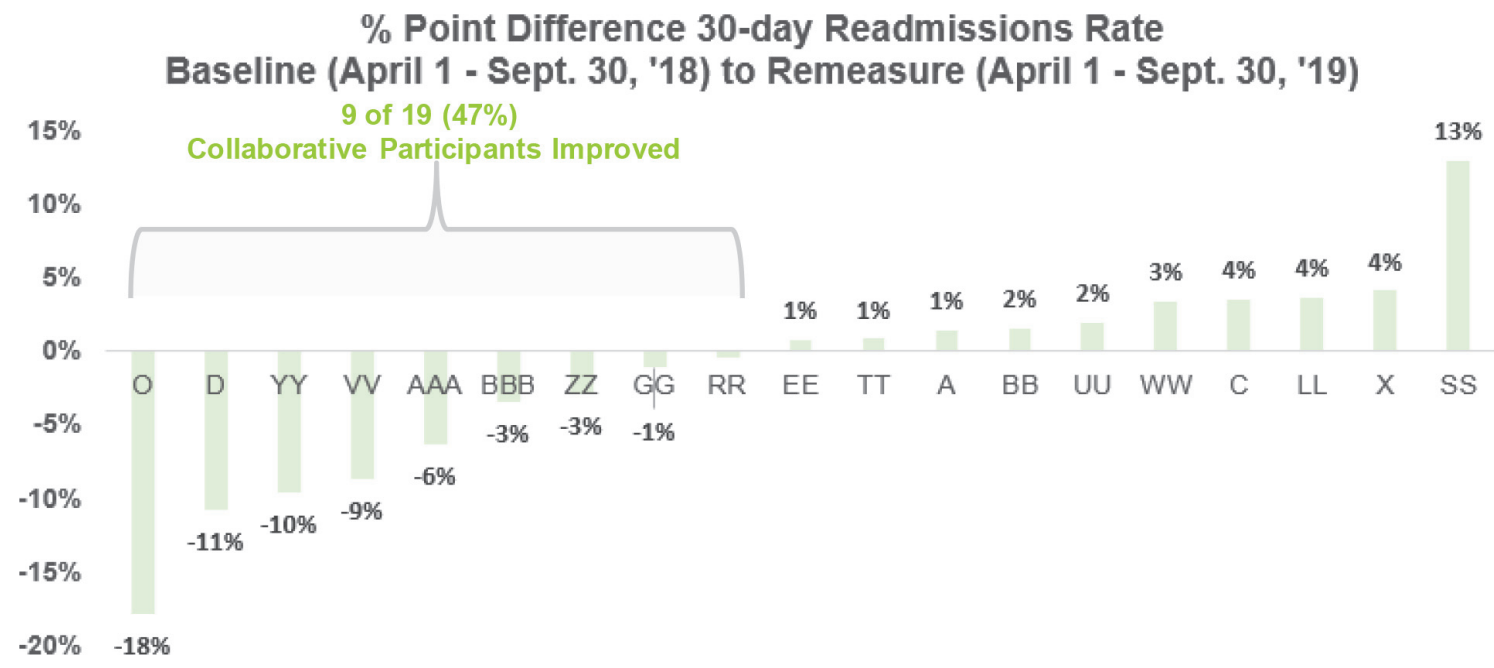

Abstract 17 Figure 3 Hemorrhagic stroke performance

\begin{tabular}{|c|c|}
\hline $\begin{array}{l}\text { Build interdisciplinary, action oriented } \\
\text { support from leadership, front line } \\
\text { champions and EMS partners }\end{array}$ & $\begin{array}{l}\text { - Regional/senior leadership support will increase buy-in, drive } \\
\text { program growth, foster clinical/operational improvements } \\
\text { - Use front line champions, a nurse and physician, to drive } \\
\text { clinical efficiencies, standardization, and alignment } \\
\text { - Prioritize strengthening partnership with EMS by understanding } \\
\text { interactions, developing and sharing education and making } \\
\text { connections }\end{array}$ \\
\hline $\begin{array}{l}\text { Standardize data collection and analysis at } \\
\text { the system level }\end{array}$ & $\begin{array}{l}\text { - Develop a meaningful reporting tool to drive change } \\
\text { management } \\
\text { - Drive adoption of reporting tool to standardize care protocols } \\
\text { and create uniformity } \\
\text { - Use reporting tool to share data with strategic partners to } \\
\text { identify priorities }\end{array}$ \\
\hline $\begin{array}{l}\text { Maintain a sense of urgency by providing } \\
\text { real-time communication }\end{array}$ & $\begin{array}{l}\text { - Implement AM and PM interdisciplinary rounds } \\
\text { - Foster friendly competitions through robust/reward recognition } \\
\text { programs | } \\
\text { - Offer continuous education opportunities for staff and partners }\end{array}$ \\
\hline $\begin{array}{l}\text { Standardize discharge planning guidelines } \\
\text { to ensure safe transitions for patients }\end{array}$ & $\begin{array}{l}\text { Targeted discharge planning guidelines to standardize: } \\
\text { - Follow-up phone call } \\
\text { - Follow-up visit } \\
\text { - Individualized care plans }\end{array}$ \\
\hline
\end{tabular}

Abstract 17 Figure 4 Key learnings for success 
two time periods. $56 \%$ reduced readmission rates for Ischemic Stroke performance and $47 \%$ reduced readmission rates for Hemorrhagic Stroke performance.

Notes (figure 1): Analysis excludes $0-17$ age group and includes the neurosciences and spine service lines and the Brain/Central Nervous System (CNS) Cancer CARE Family from the cancer service line. CNS injury includes concussion, late effects of neuro trauma, paralysis, skull fracture and major brain injury, and spinal cord injury. Movement disorders include Parkinson disease, movement disorders, multiple sclerosis and demyelinating diseases. Neuro pain disorders include headache/migraine, neuro pain and neuropathy. Other includes hydrocephalus and spina bifida, neurologic disease-other, and sleep disorders. Stroke and neurovascular include ischemic and hemorrhagic stroke, transient ischemic attack, and neurovascular diseases. Sources: Impact of Change ${ }^{\circledR}$, 2018; HCUP National Inpatient Sample (NIS). Healthcare Cost and Utilization Project (HCUP) 2015. Agency for Healthcare Research and Quality, Rockville, MD; Claritas PopFacts $^{\circledR}$, 2018; Circulation. 2019;139:e56-e528. DOI: 10.1161/CIR.0000000000000659; Sg2 Analysis, 2018.

Notes (figure 2): $n=32$. Ischemic Stroke ICD 10 Codes. Hemorrhagic Stroke ICD 10 codes. Evaluated 30 day unplanned, all cause all payer readmission rates (adults, 18 years and older). Comparisons amongst facilities is not advised since a select number of facilities met the exclusion criteria. Exclusion criteria: total discharges for at least one period was equal or less than 20 discharges. Considerations for interpreting results: volume fluctuations did occur by hospital type (primary stroke centers vs. comprehensive stroke centers), which may impact the $\%$ difference when calculating readmissions. Source: Vizient Clinical Data Base/Resource Manager ${ }^{\mathrm{TM}}$

Notes (figure 3): $N=19$. Ischemic Stroke ICD 10 Codes. Hemorrhagic Stroke ICD 10 codes. Evaluated 30 day unplanned, all cause all payer readmission rates (adults, 18 years and older). Comparisons amongst facilities is not advised since a select number of facilities met the exclusion criteria. Exclusion criteria: total discharges for at least one period was equal or less than 10 discharges. Considerations for interpreting results: volume fluctuations did occur by hospital type (primary stroke centers vs. comprehensive stroke centers), which may impact the $\%$ difference when calculating readmissions. Source: Vizient Clinical Data Base/Resource Manager ${ }^{\mathrm{TM}}$
Conclusions Key learnings for success were prioritized to reduce clinical variations in care for stroke patients and optimize system-wide transitions in care (figure 4). This collaborative model effectively identified best practices to reduce variations in stroke care while preparing for emerging advancements in technology, guidelines and new care delivery payment models.

\section{UTILIZING LEAN SIX SIGMA AND QUALITY IMPROVEMENT (QI) TO INCREASE DIABETES SELF- MANAGEMENT EDUCATION REFERRALS WITHIN THE ENDOCRINOLOGY CLINIC}

${ }^{1}$ Jessica Burks, ${ }^{1}$ Usha Kollipara, ${ }^{1}$ Jacqueline Mutz, ${ }^{1} J a s o n$ Fish, ${ }^{2}$ Estefania Andrioaia Lopez, ${ }^{2}$ Jessica Abramowitz, ${ }^{2}$ Nancy Drobycki, ${ }^{2}$ Sadia Ali. ' Southwestern Health Resources, USA; ${ }^{2}$ UT Southwestern Medical Center, USA

\subsection{6/bmjoq-2020-IHI.18}

Background Diabetes self-management education (DSME) is the ongoing process of facilitating the knowledge, skill, and ability necessary for diabetes self-care. Despite evidence that DSME improves clinical outcomes, health status, and quality of life for persons with diabetes referrals to DSME continue to be low in our population.

Objectives To increase referrals to Diabetes Self-Management Education (DSME) program from 26\% at baseline to at least $40 \%$ in 12 months in our Endocrinology Clinic.

Methods We utilized Six Sigma DMAIC (Define-Measure-Analyze-Improve-Control) methodology to problem solve and perform process improvement. We created a multi-functional team including clinic staff, providers and quality improvement personnel to assist in the design and development of the new processes. In define phase we generated a project charter and communication plan. During the measure phase we determined the baseline referral volume, completed a current state process map and completed measurement system analysis. During the analyze phase we brainstormed reasons for low referrals (root cause analysis) and analyzed referral volume for trends. During improve phase we finalized future state workflow, implemented pre-visit planning, standing medical orders for referrals and provided training to clinic staff and 